\title{
miR-328a-3p stimulates endothelial cell migration and tubulogenesis
}

\author{
SAILING CHEN $^{1 *}, \mathrm{JUN}_{\mathrm{ZHU}}{ }^{2 *}, \mathrm{YUNSONG} \mathrm{ZHANG}^{1}, \mathrm{XIAODONG} \mathrm{CAI}^{1}, \mathrm{SHENG} \mathrm{YI}^{1}$ and XINGHUI WANG ${ }^{1}$ \\ ${ }^{1}$ Key Laboratory of Neuroregeneration of Jiangsu and Ministry of Education, Co-innovation Center of Neuroregeneration, \\ NMPA Key Laboratory for Research and Evaluation of Tissue Engineering Technology Products, Nantong University; \\ ${ }^{2}$ Department of Thoracic Surgery, Affiliated Hospital of Nantong University, Nantong, Jiangsu 226001, P.R. China
}

Received January 18, 2021; Accepted July 6, 2021

DOI: $10.3892 /$ etm.2021.10538

\begin{abstract}
Endothelial cells have important biological roles after peripheral nerve injury by forming blood vessels within the nerve gap and guiding Schwann cell migration. MicroRNAs (miRNAs/miRs) affect cellular behavior and regulate a wide variety of physiological and pathological activities, including peripheral nerve regeneration. Emerging studies have identified the essential roles of miRNAs in the phenotype modulation of Schwann cells, while the effects of miRNAs on endothelial cells have remained to be thoroughly investigated. miR-328a-3p was differentially expressed in peripheral nerve stumps after nerve injury. In the present study, the effects of miR-328a-3p on biological functions of endothelial cells were determined by transfecting cultured human umbilical vein endothelial cells (HUVECs) with miR-328a-3p mimics or inhibitor. Transfection with miR-328a-3p mimics led to slightly decreased HUVEC proliferation and robustly increased HUVEC migration and tubulogenesis, while transfection with miR-328a-3p inhibitor led to opposite results. Using bioinformatics analysis, potential regulators and effectors of miR-328a-3p were further discovered and a miR-328a-3p-centered competing endogenous RNA network was constructed. Collectively, the present study demonstrated that dysregulated miR-328a-3p after peripheral nerve injury may affect the migration and angiogenesis of endothelial cells and contribute to peripheral nerve regeneration.
\end{abstract}

Correspondence to: Professor Sheng Yi or Dr Xinghui Wang, Key Laboratory of Neuroregeneration of Jiangsu and Ministry of Education, Co-innovation Center of Neuroregeneration, NMPA Key Laboratory for Research and Evaluation of Tissue Engineering Technology Products, Nantong University, 19 Qixiu Road, Nantong, Jiangsu 226001, P.R. China

E-mail: syi@ntu.edu.cn

E-mail: xhwang@ntu.edu.cn

${ }^{*}$ Contributed equally

Key words: endothelial cells, miR-328, migration, angiogenesis, peripheral nerve repair

\section{Introduction}

Endothelial cells are highly plastic cells that may rapidly switch to an activated state to mediate angiogenesis during wound healing $(1,2)$. Formed blood vessels supply oxygen and nutrients, remove wastes and contribute to the regeneration of various tissues and organs (3). Considering the importance of blood supply, vascularization is regarded as an essential factor during the fabrication and construction of tissue-engineered products (4-6).

Angiogenesis is also critical for the regeneration of injured peripheral nerves. Following peripheral nerve injury, polarized blood vessel formation within the bridge guides the migration of Schwann cells towards and across the injured site and encourages axon elongation and nerve repair (7). In tissue-engineered nerve grafts, sufficient angiogenesis benefits cell survival, promotes cell integration with biomaterials, provides a favorable nutritional microenvironment and improves the regeneration and functional recovery of injured nerves $(8,9)$. Therefore, investigating certain approaches to modulate the phenotype of endothelial cells and to advance angiogenesis after peripheral nerve injury is important in theoretical and practical aspects.

MicroRNAs (miRNAs/miRs) are small single-strand non-coding RNAs of 22 nucleotides in length. miRNAs bind to their target mRNAs, induce mRNA degradation and/or translational repression and negatively regulate their target mRNAs $(10,11)$. A large number of miRNAs were identified to be differentially expressed in the injured peripheral nerve stumps after nerve injury (12). Differentially expressed miRNAs may affect the proliferation, migration and/or remyelination of Schwann cells and regulate the nerve regeneration process (13). However, in spite of the essential roles of endothelial cells during peripheral nerve regeneration, the regulatory effects of miRNAs on endothelial cells, as compared with Schwann cells, remain largely elusive.

miR-328 has been involved in numerous biological activities, such as cellular apoptosis (14), brown adipose tissue differentiation (15) and glucose uptake (16). Emerging studies have associated miR-328 with numerous diseases, including leukemic blasts $(17)$, atrial fibrillation $(18,19)$, intervertebral disc degeneration (20) and hepatocellular carcinoma (21). Dysregulated miR-328 was also observed in prion-induced 
neurodegeneration (22), implying that miR-328 may have important roles in nervous system diseases. Furthermore, miRNA sequencing data of rat sciatic nerve stumps after nerve transection indicated that miR-328 expression was altered after peripheral nerve injury (12), suggesting that miR-328 may be essential for peripheral nerve injury and regeneration.

miR-328a-3p was previously named as miR-328 or miR-328a according to miRbase (http://mirbase.org/). In the present study, the effects of miR-328a-3p on the biological behavior of human umbilical vein endothelial cells (HUVECs) were examined. Potential upstream long non-coding RNAs (lncRNAs) and downstream target mRNAs of miR-328a-3p were bioinformatically analyzed to reveal the regulatory cascade of miR-328a-3p after peripheral nerve injury.

\section{Materials and methods}

Cell culture. HUVECs, a gift from Dr Mi Shen's laboratory at the Co-Innovation Center of Neuroregeneration, Nantong University (Nantong, China), were seeded into cell culture flasks (Corning Inc.) and cultured in endothelial cell medium (ECM; ScienCell Research Laboratories, Inc.) containing 5\% fetal bovine serum (FBS; ScienCell Research Laboratories, Inc.), $1 \%$ endothelial cell growth supplement (ECGS; ScienCell Research Laboratories, Inc.) and $1 \%$ penicillin and streptomycin (Invitrogen; Thermo Fisher Scientific, Inc.) in a humidified atmosphere with $5 \% \mathrm{CO}_{2}$ at $37^{\circ} \mathrm{C}$. The cell culture medium was refreshed every other day.

Cell transfection. Cultured HUVECs were transfected with chemically synthesized miR-328a-3p mimics, mimics control, miR-328a-3p inhibitor or inhibitor control sequences (Guangzhou RiboBio Co., Ltd.) using Lipofectamine ${ }^{\circledR}$ RNAiMAX transfection reagent (Invitrogen; Thermo Fisher Scientific, Inc.) for $48 \mathrm{~h}$ at $37^{\circ} \mathrm{C}$ according to the manufacturer's protocol. The sequences of miR-328a-3p mimics were 5'-CUGGCCCUCUCUGCCCUUCCGU-3' and 3'-ACGGAA GGGCAGAGAGGGCCAG-5', the sequence of miR-328a-3p inhibitor was 5'-ACGGAAGGGCAGAGAGGGCCAG-3', the sequences of mimics control were 5'-UUUGUACUACAC AAAAGUACUG-3' and 3'-AAACAUGAUGUGUUUUCA UGAC-5', and the sequence of inhibitor control was 5'-CAG UACUUUUGUGUAGUACAAA-3'. HUVECs were transfected with $50 \mathrm{nM}$ miRNA mimics and $250 \mathrm{nM}$ miRNA inhibitor prior to functional investigations.

Animal surgery and tissue preparation. A total of 20 6- to 8-week adult male Sprague-Dawley rats, weighing 180-220 g, were obtained from the Experimental Animal Center of Nantong University. The rats were maintained in standard laboratory conditions $\left(23 \pm 2^{\circ} \mathrm{C}\right.$ room temperature, $55 \pm 5 \%$ relative humidity and a 12-h light/dark cycle) with free access to food and water. All rats were anesthetized by intraperitoneal injection of complex narcotics $(85 \mathrm{mg} / \mathrm{kg}$ of trichloroacetaldehyde monohydrate, $42 \mathrm{mg} / \mathrm{kg}$ of magnesium sulfate and $17 \mathrm{mg} / \mathrm{kg}$ of sodium pentobarbital) (23). The rat sciatic nerve was exposed through an incision on the lateral aspect of the mid-thigh of the left hind limb. A $3 \mathrm{~mm}$ long segment of sciatic nerve was crushed two times ( 15 sec each time, 3 -sec interval) using hemostatic forceps. To minimize the discomfort and possible painful mechanical stimulation, the rats were housed in large cages with sawdust bedding post-surgery. Under anesthesia with compound anesthetic, the previously crushed sciatic nerve with both nerve ends ( $1 \mathrm{~mm}$ long) was harvested at $0 \mathrm{~h}$ and on days 1, 4, 7 and 14 after nerve crush and animals were then immediately euthanized by cervical dislocation. All experimental and animal handling procedures were performed according to the Institutional Animal Care Guidelines of Nantong University and all animal experiments were ethically approved by the Administration Committee of Experimental Animals, Nantong University (Nantong, China).

$R N A$ isolation and reverse transcription-quantitative (RT-q) $P C R$. Total RNA was extracted using TRIzol ${ }^{\circledR}$ (Thermo Fisher Scientific, Inc.) according to the manufacturer's protocol. The contaminating DNA was removed using RNeasy spin columns (Qiagen $\mathrm{GmbH}$ ). The quality of the isolated RNA samples was evaluated using an Agilent Bioanalyzer 2100 (Agilent Technologies, Inc.) and the quantity of RNA samples was determined using a NanoDrop ND-1000 spectrophotometer (Thermo Fisher Scientific, Inc.). To determine miR-328a-3p expression, a total amount of $20 \mathrm{ng}$ RNAs was reversely transcribed using a TaqMan ${ }^{\circledR}$ MicroRNA Reverse Transcription kit (Applied Biosystems; Thermo Fisher Scientific, Inc.) and subjected to RNA amplification using the QuantiNova SYBR Green PCR kit (Qiagen $\mathrm{GmbH}$ ) on an Applied Biosystems StepOne real-time PCR system. Bulge-loop miRNA qRT-PCR Primer Sets (one RT primer and a pair of qPCR primers for each set) specific for miR-328a-3p were designed by Guangzhou RiboBio Co., Ltd. RT-qPCR was performed using SYBR Green Premix Ex Taq (Takara Bio, Inc.) with primers of lncRNAs (Table S1) on an Applied Biosystems StepOne real-time PCR system to evaluate the expression abundances of the lncRNAs. The thermocycling program was as follows: $10 \mathrm{~min}$ at $95^{\circ} \mathrm{C} ; 40$ cycles of $2 \mathrm{sec}$ at $95^{\circ} \mathrm{C} ; 20 \mathrm{sec}$ at $60^{\circ} \mathrm{C}$ and $10 \mathrm{sec}$ at $70^{\circ} \mathrm{C}(24)$. The abundance of miR-328a-3p in HUVECs was compared with the internal control U6 and quantification of miR-328a-3p was performed using the $2^{-\Delta \Delta C \mathrm{C}}$ method (23).

5-Ethynyl-2'-deoxyuridine (EdU) proliferation assay. A total of $4 \times 10^{3}$ HUVECs were resuspended in $100 \mu \mathrm{l}$ culture medium and seeded onto poly-L-lysine-coated 96-well plates at a density of $4 \times 10^{4}$ cells $/ \mathrm{ml}$. HUVECs were exposed to $100 \mu \mathrm{M}$ EdU for $2 \mathrm{~h}$ and then fixed with $4 \%$ paraformaldehyde at $25^{\circ} \mathrm{C}$ for $15 \mathrm{~min}$. Cell proliferation was determined by using a Cell-Light EdU DNA Cell Proliferation Kit (Guangzhou RiboBio Co., Ltd.) following the manufacturer's protocol. Images were acquired using a DMR fluorescence microscope (Leica Microsystems). The numbers of EdU-positive cells and total cells were determined by Apollo 567 fluorescent dyes and Hoechst 33342 staining. The proliferation rates of HUVECs were calculated by dividing the numbers of EdU-positive cells by the numbers of total cells.

Wound-healing assay. HUVECs were seeded onto a mold chamber with a $1 \mathrm{~mm}$ wide insert placed on the bottom of a 6-well plate and grown in ECM medium containing 2.5\% FBS, $1 \%$ ECGS and $1 \%$ penicillin and streptomycin. After the cells had grown confluent, the insert was removed, leaving a blank 
A

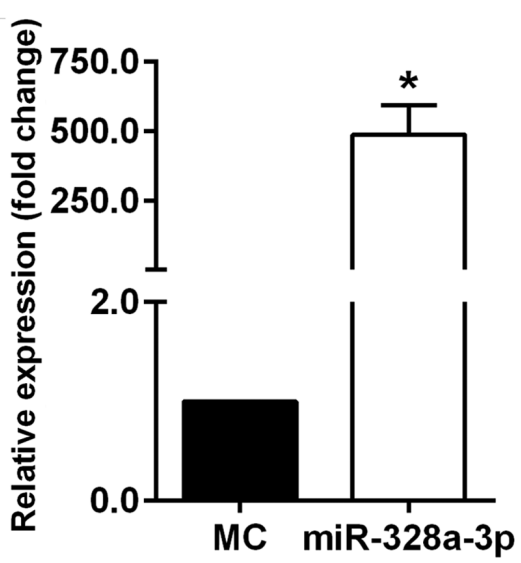

B

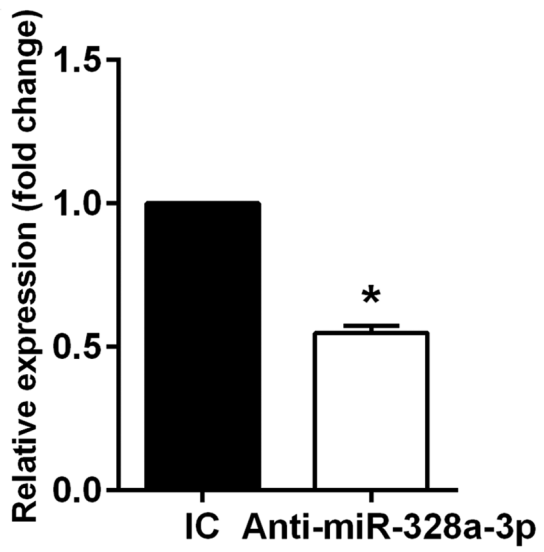

Figure 1. Transfection efficiency of miR-328a-3p mimics or inhibitor. (A) Transfection with miR-328a-3p mimics, as compared with transfection with MC, increased the relative abundance of miR-328a-3p. (B) Transfection with Anti-miR-328a-3p, as compared with transfection with IC, decreased the relative abundance of miR-328a-3p. Values are expressed as the mean with standard error of the mean from 3 experiments. ${ }^{*} \mathrm{P}<0.05$ vs. control. miR, microRNA; MC, mimics control; Anti-miR-328a-3p, miR-328a-3p inhibitor; IC, inhibitor control.

space. Images were acquired using a DMR inverted microscope (Leica Microsystems) at 0 and $12 \mathrm{~h}$ after removal of the placed insert. The relative gap closure was measured at these time-points using Image-Pro Plus 6.0 (Media Cybernetics, Inc.) from randomly selected image fields.

Transwell migration assay. A total of $1 \times 10^{4}$ HUVECs were resuspended in $100 \mu \mathrm{l} \mathrm{ECM}$ medium and seeded onto the upper compartment of a $6.5 \mathrm{~mm}$ Transwell chamber with $8 \mu \mathrm{m}$ pores (Costar; Corning Inc.) at a density of $1 \times 10^{5}$ cells $/ \mathrm{ml}$. A total of $500 \mu \mathrm{l}$ cell culture medium (ECM medium containing $2.5 \%$ FBS) was added to the lower compartment of the Transwell chamber and the HUVECs were cultured for $24 \mathrm{~h}$. The upper surface of the upper chamber was wiped with a cotton swab and the bottom surface of the upper chamber was stained with $0.1 \%$ crystal violet at $25^{\circ} \mathrm{C}$ for $15 \mathrm{~min}$. Images were acquired using a DMR inverted microscope (Leica Microsystems). The crystal violet-stained cells were dissolved in 33\% acetic acid and the optical densities of the solutions were measured at $570 \mathrm{~nm}$ (Agilent Technologies, Inc.).

Matrigel tubulogenesis assay. HUVECs were seeded onto cell culture plates pre-coated with Matrige ${ }^{\circledR}$ (BD Biosciences) and incubated for $6 \mathrm{~h}$ to allow the formation of tubules. Images were acquired using a DMR inverted microscope (Leica Microsystems). The numbers of nodes, meshes and branches in the formed tubules were quantitated using the Angiogensis analyzer in ImageJ software v1.8.0 (National Institutes of Health) (25).

Bioinformatics analysis. Upstream regulatory lncRNAs of miR-328a-3p were predicted using TargetScan (http://www.targetscan.org/vert_71/). Downstream target mRNAs of miR-328a-3p were predicted using miRWalk 3.0 (http://mirwalk.umm.uni-heidelberg.de/), miRanda (http://www.microrna.org/microrna/home.do) and the miRNA Target Prediction Database (miRdb; http://mirdb.org/). Upstream lncRNAs and downstream mRNAs were linked to construct the miR-328a-3p-centered competing endogenous
RNA (ceRNA). Heatmaps of lncRNAs and mRNAs were generated using meV software 4.9.0 (http://www.tm4.org/) according to previously obtained sequencing data of sciatic nerve stumps at $0,1,4,7$ and 14 days after nerve crush injury was described in the aforementioned protocol (26). Sequencing data were deposited in the National Center for Biotechnology Information database with the accession number PRJNA394957 (SRP113121) (27).

Statistical analysis. An unpaired Student's t-test was applied for comparisons of data between two groups. One-way ANOVA was applied for comparisons of data among multiple groups. The calculation of P-values and the generation of graphs were performed with SigmaPlot v 14.0 (Systat Software, Inc.).

\section{Results}

miR-328a-3p inhibits the proliferation of HUVECs. HUVECs were cultured and transfected with the mimics or inhibitor of miR-328a-3p to examine the effects of miR-328a-3p on endothelial cells. Efficient up- or downregulation of miR-328a-3p in cultured HUVECs with miR-328a-3p mimics or inhibitor was confirmed by RT-qPCR analysis. Transfection of HUVECs with miR-328a-3p mimics significantly increased miR-328a-3p expression (Fig. 1A), while transfection of HUVECs with miR-328a-3p inhibitor significantly decreased miR-328a-3p expression (Fig. 1B) as compared with that in their respective negative controls.

The EdU proliferation assay was performed to determine the biological effects of miR-328a-3p on the proliferation of HUVECs. The amount of proliferating cells in HUVECs transfected with miR-328a-3p mimics, as compared with that in HUVECs transfected with mimics control, was lower, while the amount of total cells in both groups was similar. The ratio of proliferating cells to total cells was lower after transfection with miR-328a-3p mimics (Fig. 2A). On the other hand, HUVECs transfected with miR-328a-3p inhibitor had an increased amount of proliferating cells and a significantly increased proliferation ratio (Fig. 2B). These outcomes 
A

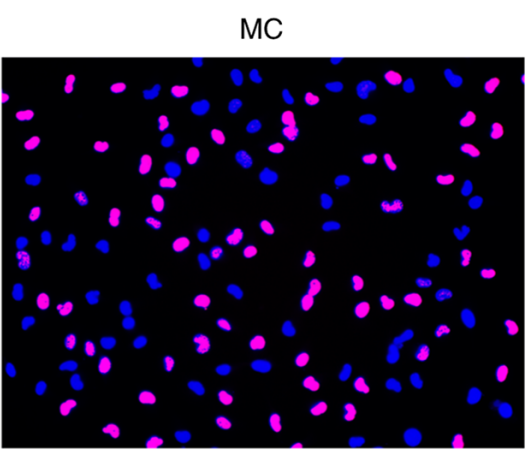

B
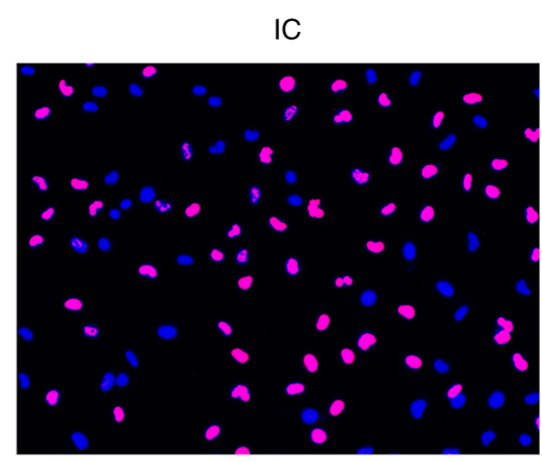

miR-328a-3p

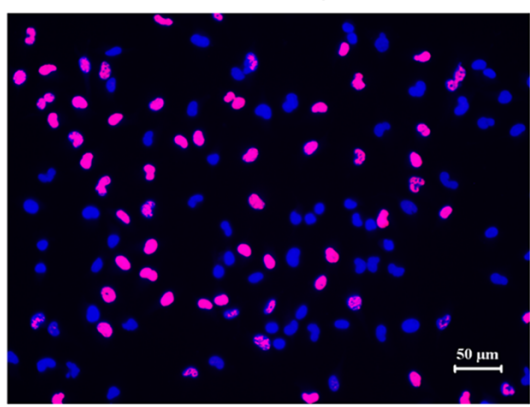

Anti-miR-328a-3p

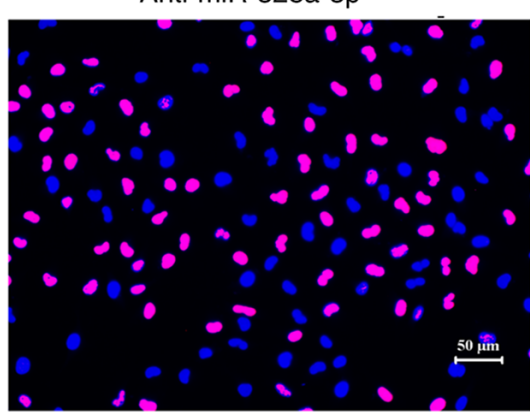

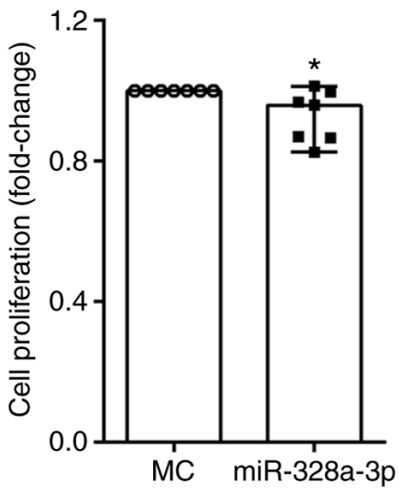

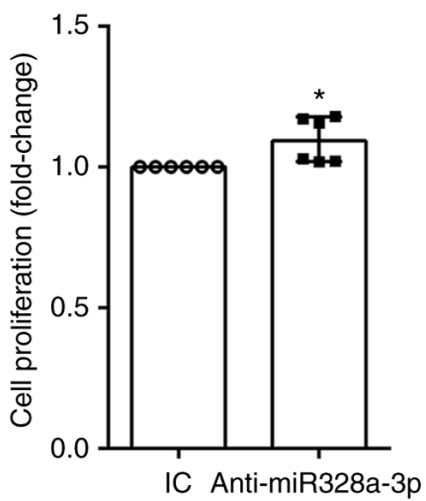

Figure 2. Effect of miR-328a-3p on human umbilical vein endothelial cell proliferation. (A) Transfection with miR-328a-3p mimics (miR-328a-3p), as compared with transfection with MC, decreased the cell proliferation rate. (B) Transfection with miR-328a-3p inhibitor (Anti-miR-328a-3p), as compared with transfection with IC, increased the cell proliferation rate. Pink color indicates 5-ethynyl-2'-deoxyuridine staining and blue color indicates Hoechst 33342 staining (scale bar, $50 \mu \mathrm{m}$ ). Data were displayed in a scatter plot as the median with interquartile range from 3 experiments using duplicate or triplicate wells. ${ }^{*} \mathrm{P}<0.05$ vs. control. miR, microRNA; MC, mimics control; Anti-miR-328a-3p, miR-328a-3p inhibitor; IC, inhibitor control.

indicated that miR-328a-3p slightly impaired the proliferation of endothelial cells.

miR-328a-3p promotes the migration of HUVECs. HUVECs transfected with miR-328a-3p mimics or inhibitor were seeded onto mold chambers with inserts placed to examine cell migration abilities. Equally wide blank spaces and same sizes of cleared area were presented after insert removal $(0 \mathrm{~h})$ for both HUVECs transfected with miR-328a-3p mimics and mimics control. Obvious cell migration was detected at $12 \mathrm{~h}$ after insert removal. In HUVECs transfected with mimics control, numerous cells migrated towards the wound area and filled the blank space. Approximately $60 \%$ of cleared area remained after $12 \mathrm{~h}$ of insert removal and cell culture, while $\sim 40 \%$ of cleared area was covered by migrated HUVECs (Fig. 3A). HUVECs transfected with miR-328a-3p mimic exhibited a relatively greater migration ability and occupied a larger space. In HUVECs transfected with miR-328a-3p mimics, the remaining cleared area was significantly smaller and only $\sim 50 \%$ of cleared area was left after $12 \mathrm{~h}$ of cell culture (Fig. 3A). In contrast to the decrease of relative cleared areas observed by miR-328a-3p mimics transfection, miR-328a-3p inhibitor transfection increased relative cleaned areas as compared with the control group (Fig. 3B).

In addition to the wound-healing assay, a Transwell assay was also applied to determine the effect of miR-328a-3p on HUVEC migration. Transfection with miR-328a-3p mimics increased the amount of migrated HUVECs (stained with crystal violet) to nearly 2 -folds of that in the mimics control group (Fig. 4A), while transfection with miR-328a-3p inhibitor reduced HUVEC migration, with a $31.07 \%$ decrease as compared with the inhibitor control group (Fig. 4B).

miR-328a-3p promotes tubulogenesis of HUVECs. HUVECs were seeded on Matrigel-coated culture plates to examine their abilities to form capillary-like tubes. HUVECs transfected with miR-328a-3p mimics, miR-328a-3p inhibitor and corresponding non-targeting negative controls were all able to form interconnecting tube networks (Fig. 5A and B). Summarized data from skeletonized images (Fig. 5C) indicated that transfection with miR-328a-3p mimics largely increased the number of formed tubes, with a $55.37 \%$ increase in the number of nodes (Fig. 5E), a $34.97 \%$ increase in the number of meshes (Fig. 5F); the increase number of branches was not significant (Fig. 5G) as compared with the mimics control. Curtailed formation of capillary-like tubes was observed in HUVECs transfected with miR-328a-3p inhibitor (Fig. 5D). Transfection with miR-328a-3p inhibitor, as compared with the inhibitor control, led to a $40.63 \%$ decrease in the number of nodes (Fig. $5 \mathrm{H}$ ), a $53.10 \%$ decrease in the number of meshes (Fig. 5I) and a slight decrease in the number of branches (Fig. 5J).

Construction of miR-328a-3p-centered ceRNA network. Upstream regulators and downstream effectors of miR-328a-3p were analyzed using bioinformatics tools. Considering that rat sciatic nerve crush injury has been commonly applied as 
A
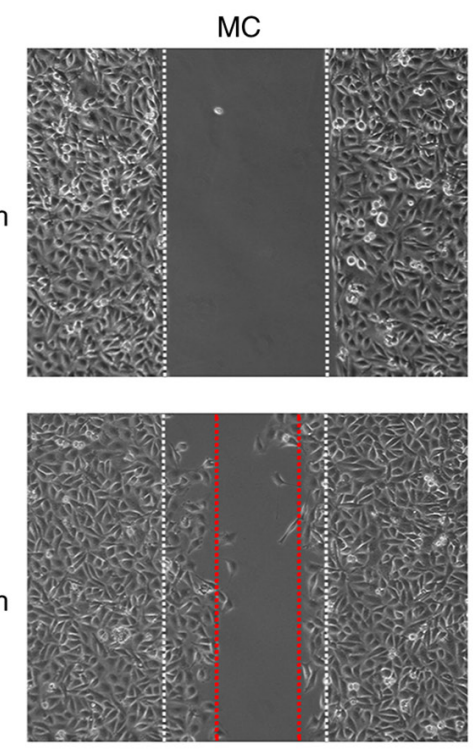

B
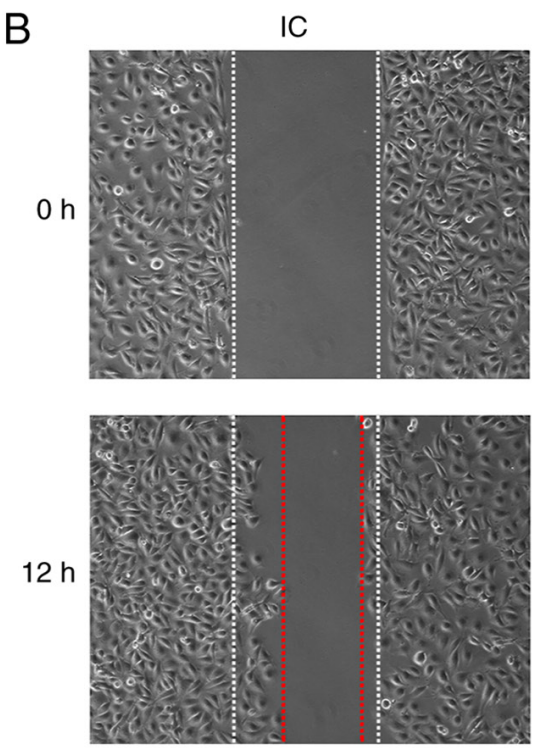
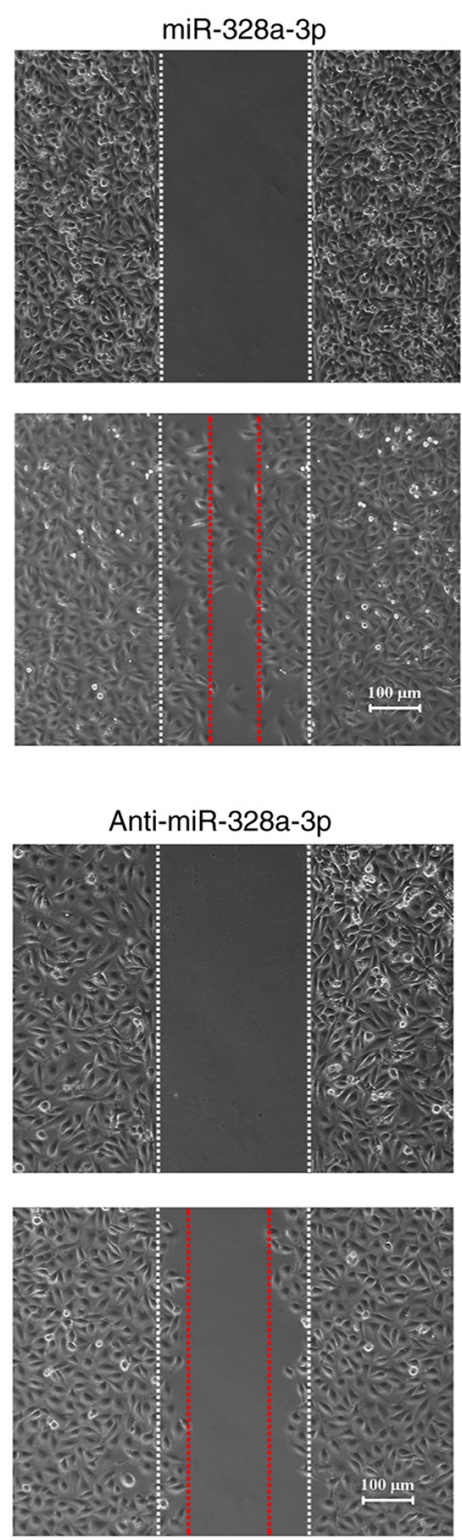
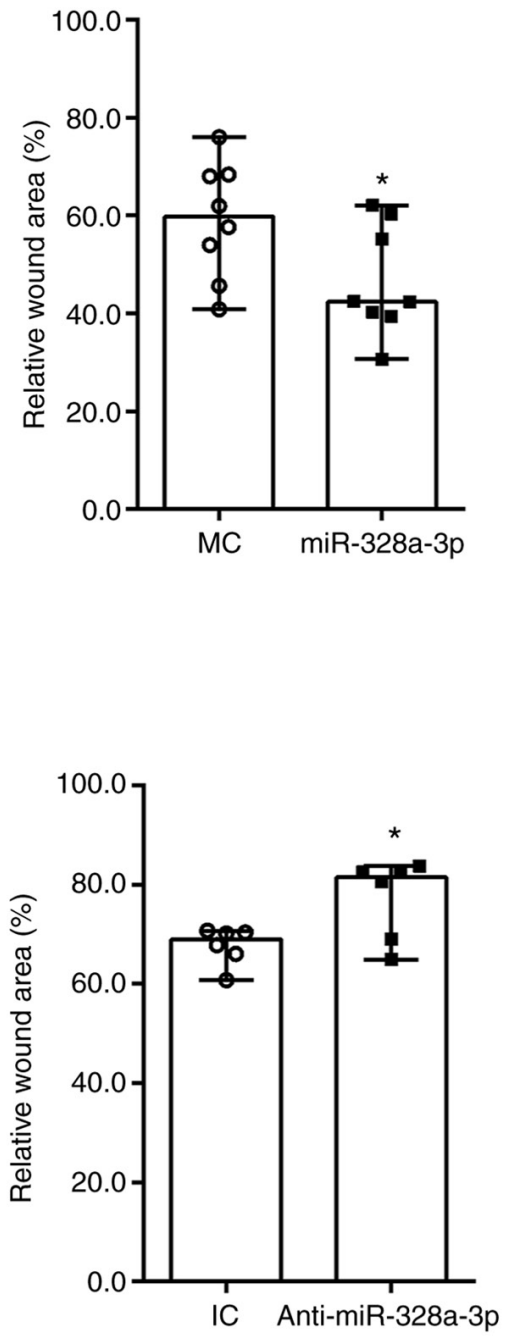

Figure 3. Effect of miR-328a-3p on wound healing of a monolayer of human umbilical vein endothelial cells. (A) Transfection with miR-328a-3p mimics (miR-328a-3p), as compared with transfection with MC, decreased the relative cleared area. (B) Transfection with miR-328a-3p inhibitor (Anti-miR-328a-3p), as compared with transfection with IC, increased relative cleared area. Dotted lines indicate the starting points of the wound and red dotted lines indicate the end points of the wound (scale bar, $100 \mu \mathrm{m}$ ). Data were displayed in a scatter plot as the median with interquartile range from 3 experiments using duplicate or triplicate wells. "P<0.05 vs. control. miR, microRNA; MC, mimics control; Anti-miR-328a-3p, miR-328a-3p inhibitor; IC, inhibitor control.

an animal model of peripheral nerve injury and gene expression profiles of rat sciatic nerve stumps were previously determined, upstream lncRNAs and downstream mRNAs of Rattus Norvegicus-miR-328a-3p were investigated. TargetScan predicted a total of 5 lncRNAs, XLOC_097278, XLOC_083369, XLOC_138151, XLOC_174539 and XLOC_053870 that are able to bind to miR-328a-3p. miRWalk 3.0, miRanda and miRdb prediction revealed various potential target genes of miR-328a-3p. From these data, an miR-328a-3p-centered lncRNA-miRNA-mRNA network was thus constructed (Fig. 6A).

Previously generated sequencing data indicated that all identified upstream lncRNAs were upregulated in sciatic nerve stumps after nerve crush injury (Fig. 6B). The results of the RT-qPCR analysis demonstrated that the expression levels of XLOC_097278, XLOC_083369, XLOC_138151,
XLOC_174539 and XLOC_053870 were elevated after sciatic nerve injury (Fig. S1). However, the majority of predicted potential target mRNAs were downregulated after nerve injury, while certain mRNAs, such as Vsig4 and Prdx5, had increased expression levels in the injured nerve stumps (Fig. 6C).

\section{Discussion}

Peripheral nerve injury and repair is a complex biological process that involves the participation of various different cell types, such as Schwann cells, fibroblasts, macrophages and endothelial cells. Following peripheral nerve injury, miRNA-mediated phenotype modulation of Schwann cells has been widely explored. However, the effects of miRNAs on endothelial cells, which are cells that have important roles in the guidance of Schwann cell migration after peripheral nerve injury, have not been well demonstrated. 
A

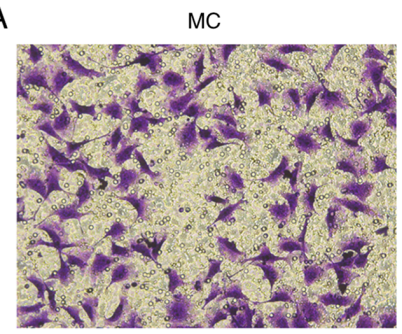

B

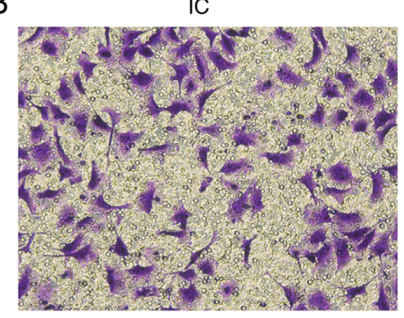

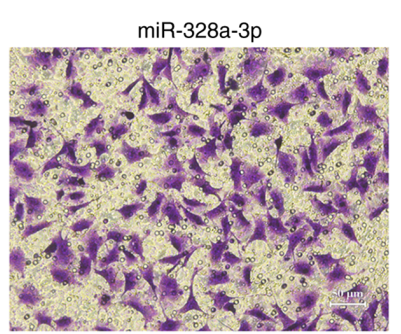

Anti-miR-328a-3p

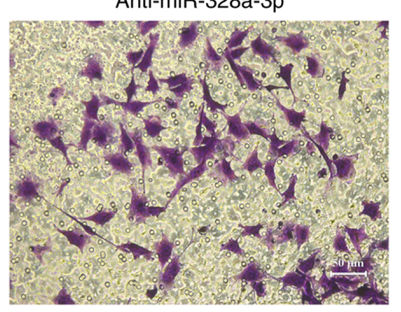

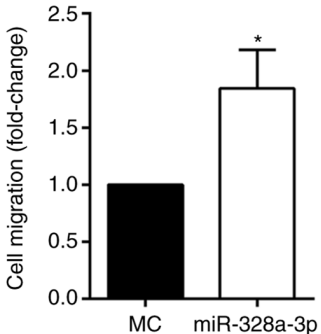

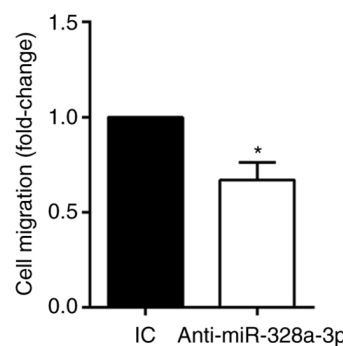

Figure 4. Effect of miR-328a-3p on human umbilical vein endothelial cell migration. (A) Transfection with miR-328a-3p mimics (miR-328a-3p), as compared with transfection with MC, increased the cell migration rate. Violet color indicated cells migrated through Transwell membranes (scale bar, $50 \mu \mathrm{m}$ ). Quantitative results were presented as the mean with standard error of the mean from 4 experiments. (B) Transfection with miR-328a-3p inhibitor (Anti-miR-328a-3p), as compared with transfection with IC, decreased the cell migration rate. Values are expressed as the mean with standard error of the mean from 3 experiments. ${ }^{*} \mathrm{P}<0.05$ vs. control. miR, microRNA; MC, mimics control; Anti-miR-328a-3p, miR-328a-3p inhibitor; IC, inhibitor control.

A

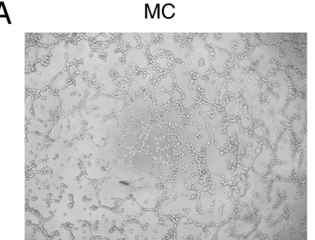

C

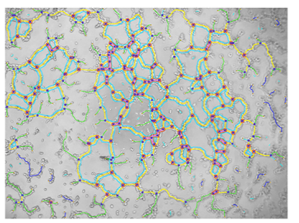

$\mathrm{E}$

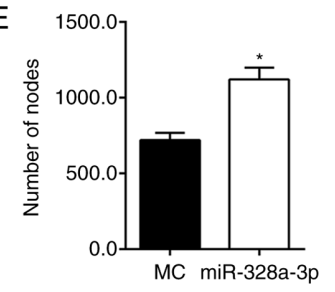

$\mathrm{H}$

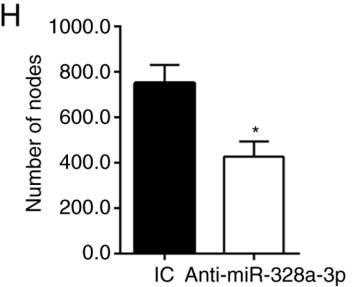

miR-328a-3p
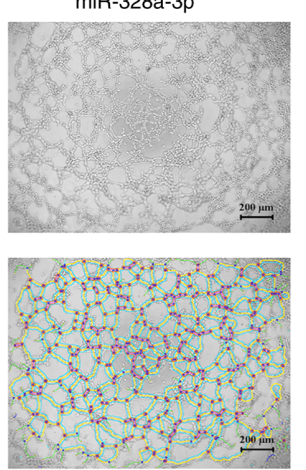

$\mathrm{B}$

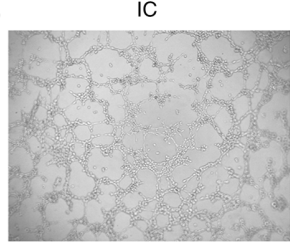

$\mathrm{D}$

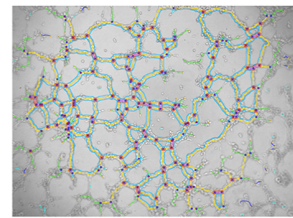

Anti-miR-328a-3p
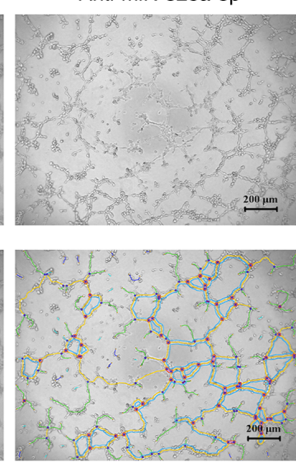

G

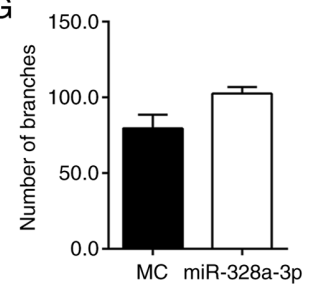

$J$

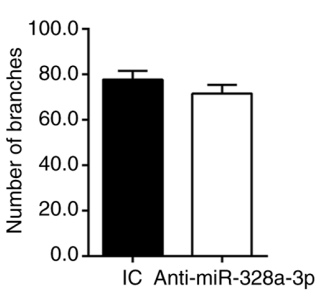

Figure 5. Effect of miR-328a-3p on tubulogenesis of HUVECs. (A) Transfection with miR-328a-3p mimics (miR-328a-3p), as compared with transfection with MC, increased cell tubulogenesis. (B) Transfection with miR-328a-3p inhibitor (Anti-miR-328a-3p), as compared with transfection with IC, decreased cell tubulogenesis (scale bar, $200 \mu \mathrm{m}$ ). (C and D) Skeletonized images of capillary-like tubes formed by HUVECs transfected with (C) miR-328a-3p mimics or mimics control and (D) miR-328a-3p inhibitor or IC. (E) The number of nodes in HUVECs transfected with miR-328a-3p mimics or mimics control. (F) The number of meshes in HUVECs transfected with miR-328a-3p mimics or mimics control. (G) The number of branches in HUVECs transfected with miR-328a-3p mimics or mimics control. (H) The number of nodes in HUVECs transfected with miR-328a-3p inhibitor or IC. (I) The number of meshes in HUVECs transfected with miR-328a-3p inhibitor or IC. (J) The number of branches in HUVECs transfected with miR-328a-3p inhibitor or inhibitor control. Values are expressed as the mean with standard error of the mean from 3 or 4 experiments. "P $<0.05$ vs. control. HUVEC, human umbilical vein endothelial cell; miR, microRNA; MC, mimics control; Anti-miR-328a-3p, miR-328a-3p inhibitor; IC, inhibitor control. 
A
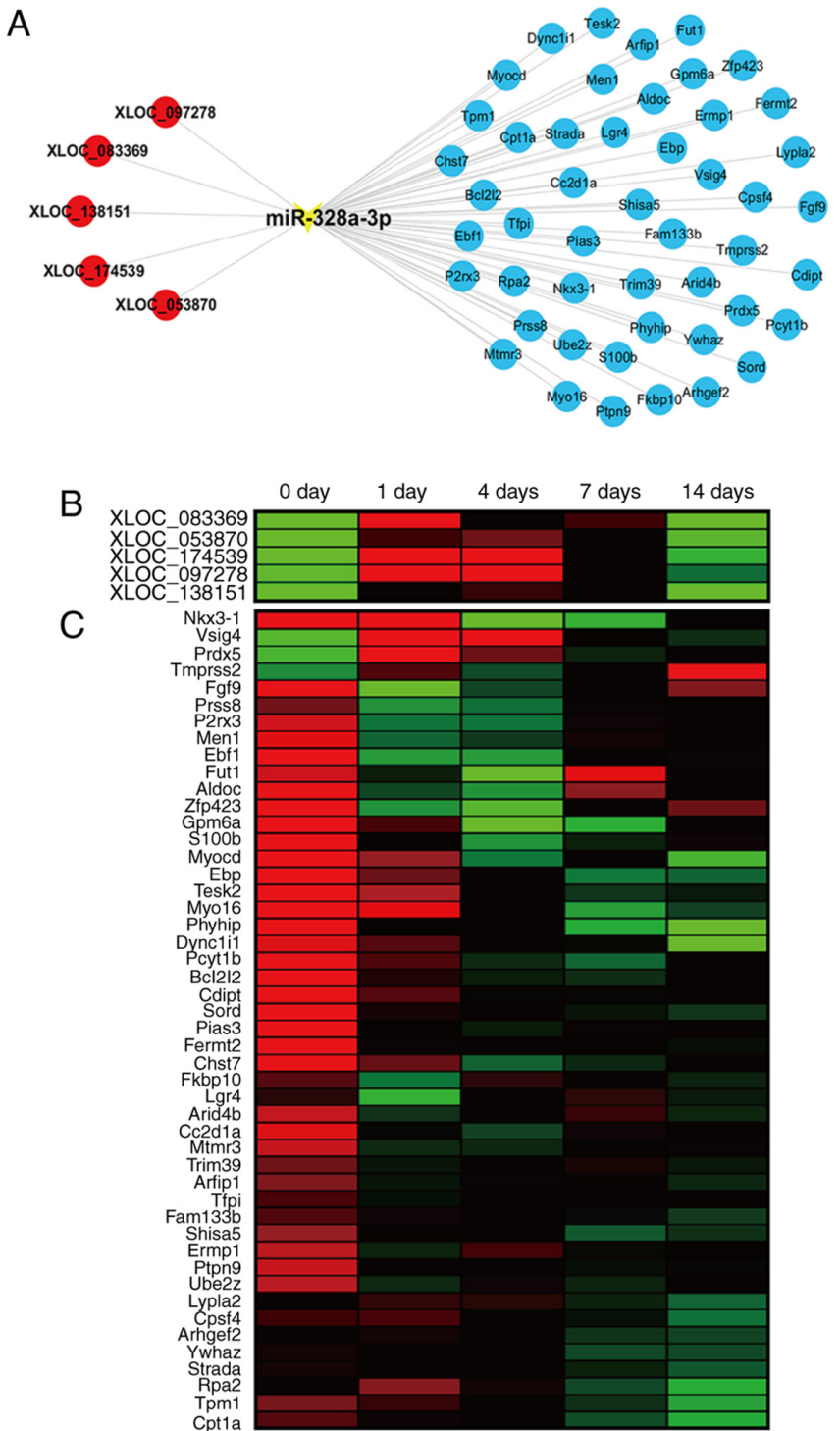

Figure 6. The ceRNA network of miR-328a-3p. (A) Interactions of lncRNAs miR-328a-3p and target mRNAs in the miR-328a-3p-centered ceRNA network. lncRNAs were labeled in red color, miRNA was labeled in yellow color and mRNAs were labeled in blue color. (B) Heatmap of the temporal expression patterns of IncRNAs in the miR-328a-3p-centered ceRNA network in the sciatic nerve stumps at $0,1,4,7$ and 14 days after nerve crush injury. Red color indicates upregulation and green color indicates downregulation. (C) Heatmap of the temporal expression patterns of mRNAs in the miR-328a-3p-centered ceRNA network in the sciatic nerve stumps at $0,1,4$, 7 and 14 days after nerve crush injury. ceRNA, competing endogenous RNA lncRNA, long non-coding RNA; miRNA, microRNA.

An earlier study by our group examined the functional effects of let-7, a well-known miRNA that belongs to the most abundant miRNA family in the genome (28), on endothelial cells (29). In the present study, the regulatory roles of miR-328a-3p, another miRNA that was demonstrated to be dysregulated in injured peripheral nerve stumps (12), were determined. The results of the wound-healing assay, Transwell migration assay and Matrigel tubulogenesis assay revealed that miR-328a-3p was able to significantly promote the migration of endothelial cells and stimulate the acquirement of functional capabilities. Considering that proliferating cells are variables of these assays, an EdU proliferation assay was also performed. In contrast to the effects of miR-328a-3p on endothelial cell migration and tubulogenesis, elevated miR-328a-3p slightly decreased EdU incorporation. These results suggested that miR-328a-3p slightly inhibits the proliferation but significantly promotes the migration of endothelial cells. Numerous regulatory factors exert consistent effects on cell proliferation and migration. However, numerous factors may have distinct roles in cell proliferation and migration. A previous study by our group indicated that MAPT, a molecule whose expression was first downregulated after nerve injury and then upregulated at later time-points, inhibits Schwann cell proliferation but encourages Schwann cell migration (30). Therefore, it is likely that miR-328a-3p has different roles to affect endothelial proliferation and migration. These results excluded the possibility that enhanced endothelial cell migration and tubulogenesis were caused by increased numbers of cells and emphasized the promoting effects of miR-328a-3p on endothelial cell migration and angiogenesis.

The functional roles of miR-328a-3p on cell migration appeared to be contradictory in various different cell types. It was reported that miR-328 functions as a tumor suppressor gene to inhibit cell migration of gastric cancer cells (31) and osteosarcoma cells (32). On the contrary, in breast cancer cells, elevated miR-328-3p was able to decrease the abundance of glutamate metabotropic receptor 4 (GRM4) and counteract GRM4-induced inhibition of breast cancer cell migration and invasion (33). Of note, various studies suggested that miR-328a-3p may even lead to different cellular behaviors in endothelial cells exposed to diverse culture conditions. Under high-glucose and low-serum conditions, the migration and tube-like structure formation of HUVECs were decreased, while the expression of miR-328 was increased. Transfection of high-glucose and low-serum-cultured HUVECs with miR-328 inhibitor stimulated cell migration and angiogenesis (14). Another study suggested that miR-328-3p promoted HUVEC migration and invasion and protected HUVECs against oxidized low-density lipoprotein-induced injury (34). These conflicting observations implied that miR-328 may have diverse functions in different microenvironments. In the present study, the biological roles of miR-328a-3p in HUVECs cultured under standardized conditions were examined. However, peripheral nerve injury generally induced a hypoxia response at the injury site $(7,35)$. In future studies, it may be helpful to mimic the pathological conditions of peripheral nerve injury, expose miR-328a-3p-treated HUVECs to decreased oxygen levels and determine the modulating effects of miR-328a-3p on the HUVEC phenotype under low oxygen levels.

Besides the functional investigations of miR-328a-3p, lncRNAs and mRNAs that may be associated with miR-328a-3p were also analyzed. The sequences of miR-328a-3p were highly conserved in different species, including human (H. Sapiens) and rat (R. Norvegicus). Considering that the transcriptome profiles were obtained and ceRNA networks of rats after peripheral nerve injury were identified (36), the present study determined lncRNA-miR-328a-3p-mRNA interactions, constructed a miR-328a-3p-centered ceRNA network, and explored the temporal expression patterns of miR-328a-3p-associated lncRNAs and mRNAs in the injured sciatic nerves of rats. Following peripheral nerve injury, the expression levels of miR-328a-3p first decreased, reaching a valley level at 4 days after nerve injury, and then gradually 
recovered to the uninjured level at 14 days (12). The heatmaps indicated inverse correlations between the temporal expression profiles of miR-328a-3p and all predicted upstream lncRNAs, as well as miR-328a-3p and the potential target genes Vsig4 and Prdx5. Vsig4 encodes for a v-set and immunoglobulin domain-containing protein and has been mainly considered as a negative regulator of T-cell responses $(37,38)$. Prdx 5 encodes for peroxiredoxin 5, a member of the peroxiredoxin family of antioxidant enzymes, and has essential antioxidant roles (39). The involvement of Vsig4 and Prdx5 in the peripheral nervous system has remained to be fully elucidated, although Prdx 5 was detected to be expressed in peripheral nerves (40) and upregulated in dorsal root ganglia neurons after peripheral nerve injury (41). In the present study, the sequencing data suggested that the abundance of Vsig4 and Prdx 5 was elevated in sciatic nerve stumps after nerve injury and elevated Vsig4 and Prdx 5 may be induced by downregulation of miR-328a-3p. The binding relationships of lncRNAs and mRNAs with miR-328a-3p may be further validated by a luciferase assay and functional rescue assays.

Collectively, in the present study, the biological effects of miR-328a-3p were investigated, revealing that miR-328a-3p increased the migration and angiogenesis potential of endothelial cells. These results provided a mechanism for the regulation of endothelial cells during peripheral nerve regeneration.

\section{Acknowledgements}

Not applicable.

\section{Funding}

This study was supported by the Natural Science Foundation of Jiangsu Province, China (grant no. BK20200976), the Priority Academic Program Development of Jiangsu Higher Education Institutions (grant no. PAPD), and Postgraduate Research and Practice Innovation Program of Jiangsu Province (grant no. KYCX21_3076).

\section{Availability of data and materials}

The datasets used and/or analyzed during the current study are available from the corresponding author on reasonable request.

\section{Authors' contributions}

SY and XW conceived and designed the study. SC, YZ and $\mathrm{XC}$ performed the experiments and acquired the experimental data. SC and XW analyzed and interpreted the data. SY, XW and JZ contributed reagents, materials and analysis tools. XW, SY and JZ drafted the manuscript and revised it critically for important intellectual content. SY and XW confirm the authenticity of all the raw data. All authors read and approved the final version of the manuscript.

\section{Ethics approval and consent to participate}

Experiments were ethically approved by the Administration Committee of Experimental Animals, Nantong University (Nantong, China).

\section{Patient consent for publication}

Not applicable.

\section{Competing interests}

The authors declare that they have no competing interests.

\section{References}

1. Eelen G, de Zeeuw P, Treps L, Harjes U, Wong BW and Carmeliet P: Endothelial cell metabolism. Physiol Rev 98: 3-58, 2018.

2. Hassan M, Moghadamrad S, Sorribas M, Muntet SG, Kellmann P, Trentesaux C, Fraudeau M, Nanni P, Wolski W, Keller I, et al: Paneth cells promote angiogenesis and regulate portal hypertension in response to microbial signals. J Hepatol 73: 628-639, 2020.

3. Velazquez OC: Angiogenesis and vasculogenesis: Inducing the growth of new blood vessels and wound healing by stimulation of bone marrow-derived progenitor cell mobilization and homing. J Vasc Surg 45 Suppl A: A39-A47, 2007.

4. Rossi L, Attanasio C, Vilardi E, De Gregorio M and Netti PA: Vasculogenic potential evaluation of bottom-up, PCL scaffolds guiding early angiogenesis in tissue regeneration. J Mater Sci Mater Med 27: 107, 2016.

5. Wong HK, Ivan Lam CR, Wen F, Mark Chong SK, Tan NS, Jerry C, Pal M and Tan LP: Novel method to improve vascularization of tissue engineered constructs with biodegradable fibers. Biofabrication 8: 015004, 2016.

6. Lloyd-Griffith C, McFadden TM, Duffy GP, Unger RE, Kirkpatrick CJ and O'Brien FJ: The pre-vascularisation of a collagen-chondroitin sulphate scaffold using human amniotic fluid-derived stem cells to enhance and stabilise endothelial cell-mediated vessel formation. Acta Biomater 26: 263-273, 2015.

7. Cattin AL, Burden JJ, Van Emmenis L, Mackenzie FE, Hoving JJ, Garcia Calavia N, Guo Y, McLaughlin M, Rosenberg LH, Quereda V, et al: Macrophage-induced blood vessels guide schwann cell-mediated regeneration of peripheral nerves. Cell 162: 1127-1139, 2015.

8. Muangsanit P, Shipley RJ and Phillips JB: Vascularization strategies for peripheral nerve tissue engineering. Anat Rec (Hoboken) 301: 1657-1667, 2018.

9. Saffari TM, Bedar M, Hundepool CA, Bishop AT and Shin AY: The role of vascularization in nerve regeneration of nerve graft. Neural Regen Res 15: 1573-1579, 2020.

10. Ambros V: The functions of animal microRNAs. Nature 431: 350-355, 2004.

11. Bartel DP: MicroRNAs: Genomics, biogenesis, mechanism, and function. Cell 116: 281-297, 2004.

12. Yu B, Zhou S, Wang Y, Ding G, Ding F and Gu X: Profile of microRNAs following rat sciatic nerve injury by deep sequencing: Implication for mechanisms of nerve regeneration. PLoS One 6: e24612, 2011.

13. Yu B, Zhou S, Yi S and Gu X: The regulatory roles of non-coding RNAs in nerve injury and regeneration. Prog Neurobiol 134: 122-139, 2015.

14. Zou Y, Wu F, Liu Q, Deng X, Hai R, He X and Zhou X: Downregulation of miRNA328 promotes the angiogenesis of HUVECs by regulating the PIM1 and AKT/mTOR signaling pathway under high glucose and low serum condition. Mol Med Rep 22: 895-905, 2020.

15. Oliverio M, Schmidt E, Mauer J, Baitzel C, Hansmeier N, Khani S, Konieczka S, Pradas-Juni M, Brodesser S, Van TM, et al: Dicerl-miR-328-Bace1 signalling controls brown adipose tissue differentiation and function. Nat Cell Biol 18: 328-336, 2016.

16. Yi W, Tu MJ, Liu Z, Zhang C, Batra N, Yu AX and Yu AM: Bioengineered miR-328-3p modulates GLUT1-mediated glucose uptake and metabolism to exert synergistic antiproliferative effects with chemotherapeutics. Acta Pharm Sin B 10: 159-170, 2020.

17. Eiring AM, Harb JG, Neviani P, Garton C, Oaks JJ, Spizzo R, Liu S, Schwind S, Santhanam R, Hickey CJ, et al: MiR-328 functions as an RNA decoy to modulate hnRNP E2 regulation of mRNA translation in leukemic blasts. Cell 140: 652-665, 2010.

18. Lu Y, Zhang Y, Wang N, Pan Z, Gao X, Zhang F, Zhang Y, Shan H, Luo X, Bai Y, et al: MicroRNA-328 contributes to adverse electrical remodeling in atrial fibrillation. Circulation 122: 2378-2387, 2010 
19. Ruan ZB, Wang F, Bao TT, Yu QP, Chen GC and Zhu L: Genome-wide analysis of circular RNA expression profiles in patients with atrial fibrillation. Int J Clin Exp Pathol 13: 1933-1950, 2020.

20. Guo W, Mu K, Zhang B, Sun C, Zhao L, Li HR, Dong ZY and Cui Q: The circular RNA circ-GRB10 participates in the molecular circuitry inhibiting human intervertebral disc degeneration. Cell Death Dis 11: 612, 2020.

21. Wei Y, Chen X, Liang C, Ling Y, Yang X, Ye X, Zhang H, Yang P, Cui X, Ren Y, et al: A Noncoding regulatory RNAs network driven by Circ-CDYL acts specifically in the early stages hepatocellular carcinoma. Hepatology 71: 130-147, 2020.

22. Saba R, Goodman CD, Huzarewich RL, Robertson C and Booth SA: A miRNA signature of prion induced neurodegeneration. PLoS One 3: e3652, 2008.

23. Wang X, Chen Q, Yi S, Liu Q, Zhang R, Wang P, Qian T and Li S: Correction: The microRNAs let-7 and miR-9 down-regulate the axon-guidance genes Ntn1 and Dcc during peripheral nerve regeneration. J Biol Chem 294: 6695, 2019.

24. Li S, Wang X, Gu Y, Chen C, Wang Y, Liu J, Hu W, Yu B, Wang Y, Ding F, et al: Let-7 microRNAs regenerate peripheral nerve regeneration by targeting nerve growth factor. Mol Ther 23 423-433, 2015.

25. Zudaire E, Gambardella L, Kurcz C and Vermeren S: A computational tool for quantitative analysis of vascular networks. PLoS One 6: e27385, 2011.

26. Yi S, Zhang H, Gong L, Wu J, Zha G, Zhou S, Gu X and Yu B: Deep sequencing and bioinformatic analysis of lesioned sciatic nerves after crush injury. PLoS One 10: e0143491, 2015

27. Zhao L and Yi S: Transcriptional landscape of alternative splicing during peripheral nerve injury. J Cell Physiol 234: 6876-6885, 2019.

28. Roush S and Slack FJ: The let-7 family of microRNAs. Trends Cell Biol 18: 505-516, 2008.

29. Ji X, Hua H, Shen Y, Bu S and Yi S: Let-7d modulates the proliferation, migration, tubulogenesis of endothelial cells. Mol Cell Biochem 462: 75-83, 2019.

30. Yi S, Liu Q, Wang X, Qian T, Wang H, Zha G, Yu J, Wang P, Gu X, Chu D and Li S: Tau modulates Schwann cell proliferation, migration and differentiation following peripheral nerve injury. J Cell Sci 132: jes222059, 2019.

31. Yan BL, Li XL and An JY: MicroRNA-328 acts as an anti-oncogene by targeting ABCG2 in gastric carcinoma. Eur Rev Med Pharmacol Sci 23: 6148-6159, 2019.
32. Shi J, An G, Guan Y, Wei T, Peng Z, Liang M and Wang Y: MiR-328-3p mediates the anti-tumor effect in osteosarcoma via directly targeting MMP-16. Cancer Cell Int 19: 104, 2019.

33. Xiao B, Chen D, Zhou Q, Hang J, Zhang W, Kuang Z, Sun Z and Li L: Glutamate metabotropic receptor 4 (GRM4) inhibits cell proliferation, migration and invasion in breast cancer and is regulated by miR-328-3p and miR-370-3p. BMC Cancer 19: 891, 2019.

34. Qin X and Guo J: MicroRNA-328-3p protects vascular endothelial cells against oxidized low-density lipoprotein induced injury via targeting forkhead box protein $\mathrm{O} 4$ (FOXO4) in atherosclerosis. Med Sci Monit 26: e921877, 2020.

35. Smaila BD, Holland SD, Babaeijandaghi F, Henderson HG, Rossi FMV and Ramer MS: Systemic hypoxia mimicry enhances axonal regeneration and functional recovery following peripheral nerve injury. Exp Neurol 334: 113436, 2020.

36. Qian T, Fan C, Liu Q and Yi S: Systemic functional enrichment and ceRNA network identification following peripheral nerve injury. Mol Brain 11: 73, 2018.

37. Jung K, Seo SK and Choi I: Endogenous VSIG4 negatively regulates the helper T cell-mediated antibody response. Immunol Lett 165: 78-83, 2015.

38. Vogt L, Schmitz N, Kurrer MO, Bauer M, Hinton HI, Behnke S, Gatto D, Sebbel P, Beerli RR, Sonderegger I, et al: VSIG4, a B7 family-related protein, is a negative regulator of $\mathrm{T}$ cell activation. J Clin Invest 116: 2817-2826, 2006.

39. Hanschmann EM, Godoy JR, Berndt C, Hudemann C and Lillig $\mathrm{CH}$ : Thioredoxins, glutaredoxins, and peroxiredoxins-molecular mechanisms and health significance: From cofactors to antioxidants to redox signaling. Antioxid Redox Signal 19: 1539-1605, 2013.

40. Lu JL, Vallat JM, Pollard JD, Knoops B and Ouvrier R: Expression of the antioxidant enzyme peroxiredoxin 5 in the human peripheral nervous system. J Peripher Nerv Syst 11: 318-324, 2006

41. Valek L, Kanngießer M, Haussler A, Agarwal N, Lillig CH and Tegeder I: Redoxins in peripheral neurons after sciatic nerve injury. Free Radic Biol Med 89: 581-592, 2015.

This work is licensed under a Creative Commons Attribution-NonCommercial-NoDerivatives 4.0 International (CC BY-NC-ND 4.0) License. 